\title{
Análisis de la situación de la futura región del Ñuble en materia de cohesión territorial
}

\author{
Rodrigo Candia
}

Universidad de Chile

\section{Resumen}

Después de un largo proceso, el proyecto de crear la región del Ñuble (en Chile) parece estar muy cerca de materializarse. Entre sus justificaciones está la situación de rezago relativo que presenta en relación al resto de la región del Biobío, lo que está ligado al concepto de cohesión territorial, definido como una condición que permite que los territorios expresen su potencial de desarrollo y ninguno de ellos queda persistentemente marginado. A partir de lo expuesto, se planteó la hipótesis de que la futura región presenta una situación de rezago ligada al concepto señalado, por lo que uno de los principales desafíos que tendrá será precisamente abordar esta condición. Del análisis realizado se pudo concluir que la zona en análisis efectivamente presenta una situación de rezago respecto a la mayoría de las regiones del país, no obstante existen condiciones favorables y oportunidades a partir del proyecto para abordarla.

Palabras clave: Cohesión, brechas, territorio.

Analysis of the situation of the future region of Nuble on territorial cohesion

\begin{abstract}
After a long process, the project to create the region Nuble (Chile) seems to be very close to materialize. Among its justifications is the situation lag relative presented in relation to the rest of the Biobio region, which is linked to the concept of territorial cohesion, defined as a condition that allows the territories to express their development potential and none of them is persistently marginalized. From the foregoing, the hypothesis that the future region presents a situation linked to lag indicated concept arose, so one of the main challenges will be precisely address this condition. The analysis it was concluded that the area actually presents a situation analysis lag compared to most regions of the country, however there are favorable conditions and opportunities from the project to address it.
\end{abstract}

Keywords: Cohesion, gaps, territory.

*Dirección de correspondencia [Correspondence

address]: Rodrigo Candia, Universidad de Chile

E-mail: racandia@gmail.com 


\section{Introducción}

Después de un largo proceso (que supera los 15 años), no exento de problemas y cuestionamientos, el proyecto de crear la región del Nuble parece estar muy cerca de materializarse.

Entre las justificaciones que han sustentado este anhelo, sin duda que está la situación de rezago relativo que presenta la actual provincia en relación principalmente al resto de la región del Biobío. Detrás de esta situación está el concepto de cohesión territorial, entendiendo como tal (en términos generales) a una condición que permite a todos los territorios que expresen su potencial de desarrollo y ninguno de ellos queda persistentemente marginado. Así, uno de los fundamentos que se ha declarado por parte de los principales promotores de esa iniciativa ha sido que permitirá abordar las desigualdades y condiciones de pobreza que aquejan a las diversas comunas que la compondrían, las que en su mayoría además tienen un alto porcentaje de población rural.

A partir de lo expuesto, este trabajo se plantea abordar la hipótesis de que la futura región presenta una situación de rezago en diversos indicadores ligados al concepto de cohesión territorial, por lo que uno de los principales desafíos que tendrá será precisamente abordar las brechas que existan en ese ámbito.

Para analizar este planteamiento, se ha definido como objetivo general precisamente el efectuar un análisis comparativo de la futura región del Ñuble, en relación a otras regiones del país, a partir del tipo de indicadores ya señalados, los cuales se definieron teniendo como referencia la literatura disponible y los datos para su análisis fueron extraídos principalmente de fuentes tales como la encuesta de caracterización socioeconómica CASEN, entre otras.

De él se desprenden los objetivos específicos de analizar los alcances de la cohesión territorial, mediante su operacionalización en indicadores que permitan su evaluación y comparación respecto a otros territorios y; formular propuestas de políticas, desde la perspectiva de cohesión territorial, que permitan abordar las brechas que sean detectadas.

Por último, con el análisis efectuado se plantearon propuestas para abordar las brechas que se detectaron, en el escenario de la puesta en marcha de la nueva región.

\section{Contexto territorial}

La actual provincia de Nuble data desde los inicios de la República, creándose el 2 de febrero de 1848. Agrupa a un total de 21 comunas, siendo una de la que más comunas poseen a nivel nacional.

En cuanto a sus características, de acuerdo al estudio "Línea Base, Consideraciones y Propuestas Técnicas para Determinar Pertinencia de Creación de Nueva Región de Nuble", efectuado por la Universidad de Concepción (UDEC) entre los años 2013 y 2014, el territorio de la actual provincia presenta las siguientes:

1. Demografía: 438.103 habitantes (censo año 2002), concentradas principalmente en Chillán y San Carlos; el $70 \%$ de los habitantes habita en localidades consideradas como pueblo, de acuerdo a lo definido por el INE, mientras que solo el $3 \%$ lo hace en ciudades mayores; La mayoría de las comunas concentran su población en zonas rurales.

2. Indicadores sociales: Presenta un 19,5\% de pobreza, mientras que en la totalidad de la región del Biobío este indicador llega a los 21,5\%. Si bien este indicador ha disminuido, siguiendo la tendencia del resto del país, en general ha sido a tasas menores que el promedio nacional; la pobreza se concentra principalmente en la zona precordillerana andina y en el secano interior de la actual provincia; a partir del porcentaje señalado, la actual provincia sería una de las regiones que presentaría tasas más altas de pobreza, siendo éstas solo mejores a las que tiene la región de La Araucanía y la restante región del Biobío.

3. Estructura económica: Presenta un índice de diversificación económica de 0.8653 , lo que la dejaría como la región menos diversificada a nivel nacional (medida por ocupados por rama), centrada principalmente en el sector agrícola; las actividades terciarias representan un $53 \%$ del total de actividades productivas que se realizan en la provincia, lo que estaría dentro de las menores participaciones a nivel nacional. Además, esas actividades se concentran en pocas comunas de la provincia.

4. Otras: El Índice de Desarrollo Humano de la región de Nuble sería de 0.5607 , la que la dejaría como la menos desarrollada a nivel nacional (dentro de la nueva región, las que presentan un mejor indicador son las comunas de Chillán Viejo, Yungay y Chillán); el coeficiente de gini sería de 0.4965 , lo que estaría bajo el promedio nacional de 0.5341 y de la cifra que 
presenta la región del Biobío actual, que llega a 0.5360; en materia de educación, se aprecia una baja en la matrícula a nivel escolar en toda la región del Biobío, lo que por tanto también se expresa en la provincia de Nuble. No obstante, la matrícula de educación superior ha aumentado, concentrándose un $15 \%$ de los estudiantes de la región en la provincia de Nuble; en cuanto a la superficie territorial, la nueva región sería la que tendría la menor de todas las regiones.

Por su parte, el proyecto de ley que crea la XVI Región de Nuble y las provincias de Diguillín, Punilla e Itata (mensaje $\mathrm{N}^{\circ} 761-363$ ) destaca, entre los antecedentes que entrega para justificar esta iniciativa, que el territorio en cuestión posee una identidad, una historia y una tradición que lo distingue. Ello por cuanto "se reconoce como una zona con tradiciones muy arraigadas, asociada a lo rural, y que ha generado un gran legado en base a su histórica producción agrícola y ganadera. Del mismo modo, también se reconoce como una parte del país dotada de un amplio patrimonio histórico y cultural; junto a un nutrido tejido de actores y organizaciones sociales" (2015; p.1).

Además, releva que las dinámicas territoriales y espaciales que ocurren en la actual provincia son muy diferentes a las que se dan en la capital de la región del Biobío. Ello se expresa, por ejemplo, en la base de sus estructuras productivas, en donde Nuble la agricultura ocupa un lugar primordial, mientras que en la capital regional destacan rubros del sector secundario como la industria manufacturera.

Por ello, agrega, la creación de la nueva región busca generar las condiciones institucionales y de recursos que permitan impulsar de forma pertinente el desarrollo de esta zona, basado también en las definiciones establecidas por sus propios habitantes.

\section{Cohesión territorial}

De acuerdo a los objetivos definidos para el presente trabajo, sin duda que el principal concepto que lo enmarca es el de cohesión territorial. Para analizar su definición e implicancias, se han revisado diversos documentos.

En el primero de ellos, elaborado por Silva y Echeverri (2015), inicialmente se expone el origen del concepto, el que según señalan se encuentra en Europa, en el marco del proceso de generación y consolidación de la comunidad de naciones, y planteó la necesidad de abordar la integración y convergencia a nivel territorial.
Detrás de él, siguiendo a ambos autores, está la idea de brechas territoriales, entendiendo como tal "a las diferencias [que existen] en la distribución de las actividades y dinámicas económicas en el espacio [, siendo] expresión de la desigualdad que se genera por los procesos de inversión, acumulación y remuneración de los diferentes componentes de la producción" (Silva y Echeverri, 2015: 27).

Así, de acuerdo con los autores señalados, en términos generales, se entiende a la cohesión territorial como un objetivo que busca lograr en simultáneo crecimiento económico, equidad social, sostenibilidad ambiental y gobernanza política que permita integrar y alcanzar un equilibrio entre territorios diversos.

Esto se complementa con la importancia que tiene la visión territorial para mejorar la calidad de las políticas públicas y de la acción pública en general, ya que (de acuerdo a los autores en análisis) permite considerar las particularidades y diferencias que no solo se expresan en condiciones para el desarrollo, sino que también en rasgos culturales y políticos que configuran sus propios proyectos de desarrollo.

Junto con el citado, otro documento revisado fue el elaborado por el ODTA $(2009)^{1}$, el cual define la cohesión territorial "como un principio para las actuaciones públicas encaminadas al logro de objetivos [tales] como crear lazos de unión entre los miembros de una comunidad territorial (cohesión social) y favorecer su acceso equitativo a servicios y equipamientos (equidad/justicia espacial), configurar un auténtico proyecto territorial común (identidad) partiendo del respeto a la diversidad y a las particularidades, articular y comunicar las distintas partes del territorio y romper las actuales tendencias hacia la polarización y desigualdad entre territorios [...] aprovechando las fortalezas y rasgos inherentes de cada uno de ellos" (ODTA, 2009:2). Una definición similar es la que entregan Tabales et al. (2009).

Luego, siguiendo el citado documento, se destaca que la cohesión territorial sería un principio de la acción pública orientada hacia el desarrollo territorial, el cual se conformaría a partir de 3 componentes, a saber:

1. Articulación física entre las partes del territorio a través de las redes de infraestructuras.

2. Equidad territorial, "entendida como la igualdad de oportunidades para alcanzar el desarrollo de la persona en todas las partes de un territorio [lo que se manifiesta] al garantizar la

\footnotetext{
${ }^{1}$ OCTA: Observatorio de la Diversidad, Cohesión y el Desarrollo Territorial de Andalucía.
} 
igualdad en los niveles de prestación de servicios públicos, equipamientos e infraestructuras en todas las partes del territorio considerado" (ODTA, 2009:2).

3. Identificación de la comunidad que habita un territorio a partir de un proyecto de vida en común.

Finalmente, el documento en análisis junto con el de Tabales et al. (2009) plantean el aporte que puede hacer el concepto de cohesión territorial a los enfoques de cohesión económica y social, señalando que:

1. Introduciría al territorio dentro de los factores que permitirían explicar las desigualdades.

2. Derivado de lo anterior y del reconocimiento de las especificidades territoriales, se podría lograr mayor eficacia y eficiencia al diseño e implementación de políticas públicas orientadas a la cohesión económica y social.

3. Además, el foco territorial puede servir de base para lograr la integración y articulación de políticas y agentes ligados a ellas, lo que siempre es difícil.

4. $\mathrm{Su}$ consecución requiere necesariamente de participación pública, lo cual se traduce en información, involucramiento y empoderamiento de los actores de cada territorio.

5. Por último, la cohesión territorial es de utilidad para generar identidad y sentido de pertenencia territorial.

Esto se complementa con lo señalado por Silva y Echeverri (2015), quienes plantean a propósito de los objetivos y componentes de la cohesión territorial, que estos "[...] no son diferentes de [los definidos] para la cohesión social y económica y que [...] se expresan en términos y componentes similares, esto es, como expresión de convergencia de los indicadores de desarrollo económico, social y ambiental" (Silva y Echevarría; p.35).

Otro documento revisado para efectos de este trabajo fue el elaborado por la CRMP $(2008)^{2}$, el que destaca el carácter político que tiene el concepto, ya que "está basado en una idea central de equidad y, por lo tanto, de cohesión entre los territorios a una escala determinada" (CRMP, 2008: 2). Ello lo plantea en el contexto de que la cohesión territorial se orientaría, en términos generales, a que los diferentes territorios aprovechen de forma equitativa

\footnotetext{
${ }^{2}$ CRMP: Conferencia de Regiones Periféricas Marítimas de Europa.
}

de los beneficios de la acción que se impulsa desde el nivel nacional, considerando que las condiciones que disponen para eso son dispares.

Relacionado a este concepto, emergen otros 2 relevantes: el de participación ciudadana, el de territorio y el de desarrollo territorial. Acerca del primero, de acuerdo con Rentería (2011), destaca que ya desde inicios de la década de los $90^{\prime}$ los informes de desarrollo humano plantean lo imprescindible que resulta impulsar mecanismos de participación para abordar diversos tipos de problemas, relevando la importancia tanto de los actores sociales como de las redes que caracterizan sus interacciones para generar e implementar procesos de desarrollo.

No obstante, luego también indica que si bien este es un tema que ha adquirido importancia y consideración, su práctica presenta una serie de problemas, tales como las limitaciones que existen sobre el alcance del concepto por parte de algunos actores del sector público, que lo restringen principalmente a entregar información a la ciudadanía; y la falta de organización en la sociedad y las dificultades que tiene el enfrentarlas, entre otras. No obstante, el mismo documento aclara que estos problemas no deben ser motivo para que no se realice participación.

Junto con ello, el documento citado existen distintos tipos de participación, los que pueden analizarse de acuerdo tanto a la dependencia gubernamental (de la que surgen las categorías de institucionalizada, no institucionalizada, autónoma, clientelista e incluyente o equitativa) como a aspectos objetivos y subjetivos, interrelaciones e incidencia sobre la realidad (clasificándose en espontánea, mecánica, reflexiva y creativa). En ese contexto, se señala que la definición del o de los tipos de participación que se desea promover depende de las características del capital social, en los valores que se apoya y en los resultados que son susceptibles de ser alcanzados a partir de ella. En ese sentido, se plantea que la participación reflexiva y la creativa son las que mejores impactos positivos tienen sobre el territorio, sin embargo, estas requieren de interrelación fuerte entre los participantes, de claridad en los objetivos a obtener, y de contar con horizontes amplios, en lo que se refiere a características del capital social; y de solidaridad, respecto, orientación hacia el bien común y de capacidad de diálogo y generar consensos, en lo que a valores se refiere.

Respecto al segundo de ellos, siguiendo a Schejtman y Berdegué (2004), es importante concebir al territorio no solo como un lugar geográfico, sino que como un sujeto activo y determinante que condiciona los procesos de desarrollo que se formulen e impulsen en él. De ahí se entiende la idea de construc- 
ción social del territorio que plantean los autores citados, la cual implica concebirlo "como un conjunto de relaciones sociales que dan origen y a la vez expresan una identidad y un sentido de propósito compartidos por múltiples agentes públicos y privados (aunque dicha construcción implique muchas veces transitar por procesos de conflicto y negociación)" (Schejtman y Berdegué, 2004: 29). Siguiendo a dichos autores, es esa identidad la que es la base de los proyectos colectivos de desarrollo, en donde confluyen objetivos, necesidades e intereses comunes.

Por su parte, el enfoque de desarrollo territorial, siguiendo a los autores ya señalados, requiere considerar ese rol protagónico del territorio en los procesos de desarrollo reconociendo, a su vez, la heterogeneidad social que presenta cada uno de ellos (y, más ampliamente, de la situación social y económico-productiva que cada uno de ellos tiene), la necesidad de promover la articulación y concertación de los actores sociales a partir de proyectos comunes de desarrollo y la disposición de una institucionalidad que permita lo anterior y el impulso del proceso de desarrollo en general. Así, focalizando el análisis en el ámbito rural, la idea de desarrollo territorial se entiende como "un proceso de transformación productiva e institucional de un espacio rural determinado, cuyo fin es reducir la pobreza rural" (Schejtman y Berdegué, 2004: 30), en donde se aprovechan las capacidades locales para alcanzar mejoras en el bienestar de las personas que son parte de ellas y se generan las condiciones necesarias para asentar y proyectar los procesos que permiten obtener tales logros.

Así, en definitiva, "la perspectiva de cohesión territorial aporta una visión espacial o territorial a los otros conceptos, trasladando todas aquellas cuestiones que antes se presentaban bajo un prisma puramente social (igualdad, equidad, solidaridad, protección social) a un enfoque territorial (solidaridad y justicia territoriales)" (ODTA, 2009: 2).

Estos planteamientos, desde el punto de vista del sustento teórico, también se ven complementados por el enfoque de política regional denominado Placed-Based Policy, el cual fue desarrollado por Barca, McCann y Rodríguez-Pose, el que tiene como uno de sus objetivos el promover la equidad territorial y orientar la acción hacia la generación de empleo y el fomento productivo en aquellos territorios que no los poseen. Esto por cuanto, siguiendo a Neumark y Simpson (2014), "los efectos de bienestar locales pueden diferir sustancialmente de los que están en un nivel agregado. La explotación de las externalidades de aglomeración en un solo lugar podría ir en detrimento de beneficios de aglomeración en otras áreas y producir distorsiones en la ubicación eficiente de la actividad económica" (14).

En virtud de lo expuesto, para efectos de este trabajo se entenderá como cohesión territorial tanto a una condición como a una capacidad local mediante la cual todas las zonas de un determinado lugar están en posición de disponer y generar niveles mínimos de bienestar, definidos como tal por la sociedad reconociendo los rasgos particulares que implican para cada sector. Para abordar las diferencias entre ellos, se deben generar intervenciones que apuntan a la convergencia territorial, las que además se deben sustentar en principios de integración y solidaridad.

\section{Metodología}

La metodología que se describe a continuación buscará obtener los antecedentes necesarios para responder a pregunta ¿en qué situación se encuentra la futura región de Nuble en materia de cohesión territorial?

Para ello, a partir de la contextualización teórica desarrollada en los puntos anteriores, se ha realizado un análisis comparativo de la situación de la actual provincia de Nuble en relación al resto de las regiones del país a partir de una serie de indicadores definidos con base en una operacionalización del concepto de Cohesión Territorial. Para esto último, se ha revisado una serie de bibliografía que entrega experiencias, recomendaciones y orientaciones de las que se han extraído las siguientes:

1. Como consideración general los indicadores a seleccionar deberán ajustarse a una serie de criterios, debiendo ser relevantes (relacionados con metas, objetivos y prioridades de política, socialmente validados como tal); consensuados (con respaldo técnico y social hasta donde sea posible); fidedignos (sustentados en datos respaldados y confiables); funcionales (que sean de utilidad para formular intervenciones que permitan mejorar las condiciones de vida); cuantificables (es decir, medibles); y comparables (tanto con otros territorios - de diverso tamaño - como con periodos de tiempo diferentes).

2. La complejidad de factores que están ligados al concepto de cohesión territorial trae consigo la necesidad de lograr su medición a través de indicadores más acotados y abordables. Por ello, "si se intenta una aproximación mediante indicadores al complejo concepto de la cohesión territorial, sería conveniente hacerlo a través 
de indicadores sintéticos [...], pudiendo practicar la medición de aspectos fragmentarios de la cohesión territorial, pero desde la perspectiva de la dificultad, posiblemente irresoluble, de mezclar cuestiones diferenciadas para expresar un concepto tan complejo como éste. Entre los elementos a tener en cuenta en su medición destacan"(ODTA, 2009: 11):

- Articulación física por redes de transporte.

- Acceso equivalente a equipamientos y servicios.

- Desarrollo económico equilibrado, es decir: armónico con el ambiente, la sociedad y las capacidades políticas de cada lugar.

- Aprovechamiento del capital territorial, entendiendo como tal a aquel que se define por las condiciones naturales y por las construidas por el hombre, y por las condiciones humanas (recursos humanos), sociales (capacidad de gobernanza) y de imagen, siendo esto último lo ligado a la identidad de cada lugar.

Esta complejidad también es reconocida por Pillet et al. (2013), quienes señalan que los indicadores que se utilicen deben necesariamente ser capaces de abordar la naturaleza multidisciplinar del concepto. En ese marco, además, proponen algunos indicadores estructurados en 3 criterios: Eficiencia territorial, Calidad territorial e Identidad territorial.

3. Algunos pasos relevantes a considerar: elaboración de los criterios de medición; medir las disparidades territoriales a partir de los indicadores seleccionados (evaluar ponderación de criterios); discusión y análisis (?).

Teniendo lo expuesto en consideración, se han definido algunos indicadores para la medición de la cohesión territorial, cuyos datos fueron extraídos de la encuesta de Caracterización Socioeconómica (CASEN) y del Sistema Nacional de Información Municipal (SINIM). Con estos indicadores se ha conformado la matriz de análisis presentada en la tabla 1:

Los principales resultados de este análisis son presentados en el punto siguiente. Luego, con base en ellos, se analizaron las brechas que presenta la actual provincia (y eventual nueva región) del Ñuble en relación al resto de las regiones para, finalmente, formular recomendaciones de política que permitan abordarlas y que, a su vez, puedan ser consideradas dentro de las acciones iniciales que podrían ejecutarse luego de que inicie el funcionamiento de la nueva región.

\section{Resultados y discusión}

La información de los indicadores identificados en el punto anterior, extraída de la base de datos de la encuesta $\mathrm{CASEN}^{4}$ y del $\mathrm{SINIM}^{5}$, arrojó los siguientes resultados ${ }^{6}$ :

\section{Indicadores sociales}

\section{Tasa de pobreza}

Tal como lo muestra la figura 1, en lo que se refiere a la tasa de pobreza (pobres no indigentes), la futura región del Nuble presenta un 14,7 \%, lo cual la deja entre más que presentan un mayor porcentaje a nivel nacional, siendo superada solo por las regiones del Biobío (en su composición futura) y de La Araucanía.

Esa pobreza, en su mayor porcentaje, es de carácter rural, lo cual tiende a ser más aguda por la mayor precariedad de condiciones que disponen estos entornos, en relación al urbano, en términos de infraestructura y servicios; entre otras razones.

\section{Tasa de indigencia}

Por su parte, en lo que se refiere a su tasa de indigencia, la situación de la nueva región empeora respecto al contexto nacional, posicionándose en el penúltimo lugar, detrás únicamente de la región de La Araucanía. Esto se refleja en la figura 2.

$\mathrm{Al}$ igual que en caso anterior, se deben considerar además los rasgos de ruralidad que presenta la zona en análisis.

\section{Nivel educacional}

Respecto al nivel educacional que presenta la actual provincia de Nuble, los antecedentes obtenidos entregan datos como los siguientes:

- Sobre el indicador "sin educación formal", se encuentra entre las zonas con mayor porcentaje de población en esa condición (lugar 11),

\footnotetext{
${ }^{4}$ Los datos fueron extraídos de la encuesta levantada el año 2011, debido a que la del año 2013 (en formato para SPSS), no dispone de las variables necesarias para elaborar la variable "región del Nuble".

${ }^{5}$ Información obtenida desde el sitio Web www.sinim.cl en noviembre de 2015.

${ }^{6}$ La variable "región del Ñuble" se construyó, en ambas bases de datos, a partir de las comunas que la compondrían. Los datos correspondientes a la región del Biobío corresponden a lo que sería la región sin la actual provincia de Nuble.
} 
Figura 1: Tasa de pobreza por región (pobres no indigentes), año 2011 (en porcentajes)

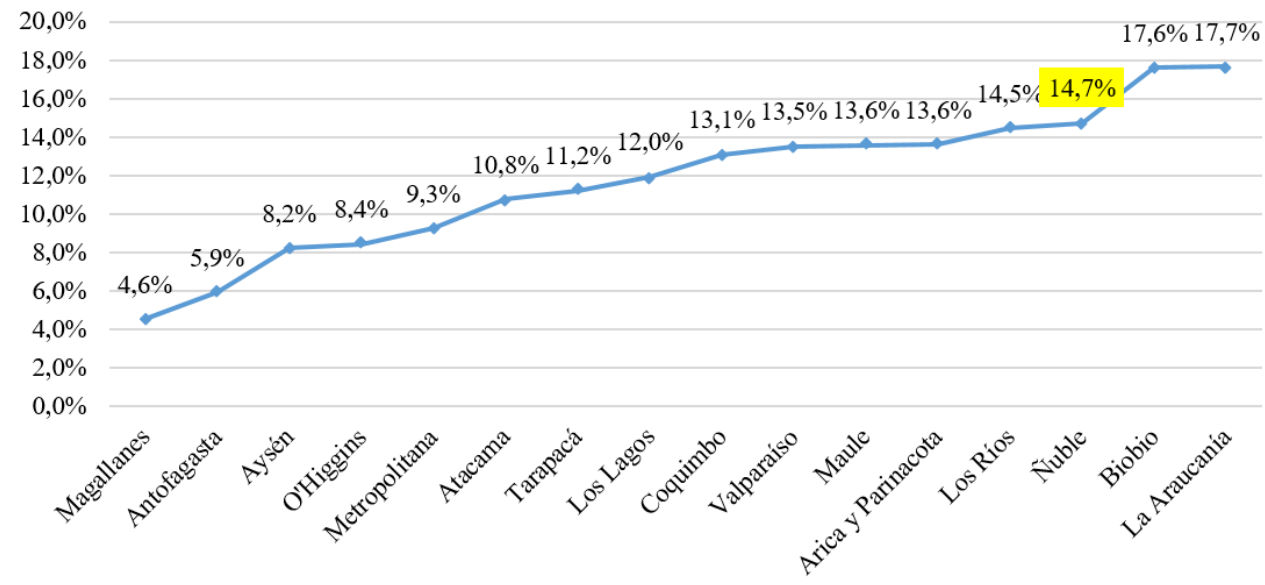

Fuente: elaboración propia con base en Encuesta CASEN 2011

Figura 2: Tasa de indigencia por región (pobres extremos), año 2011 (en porcentajes)

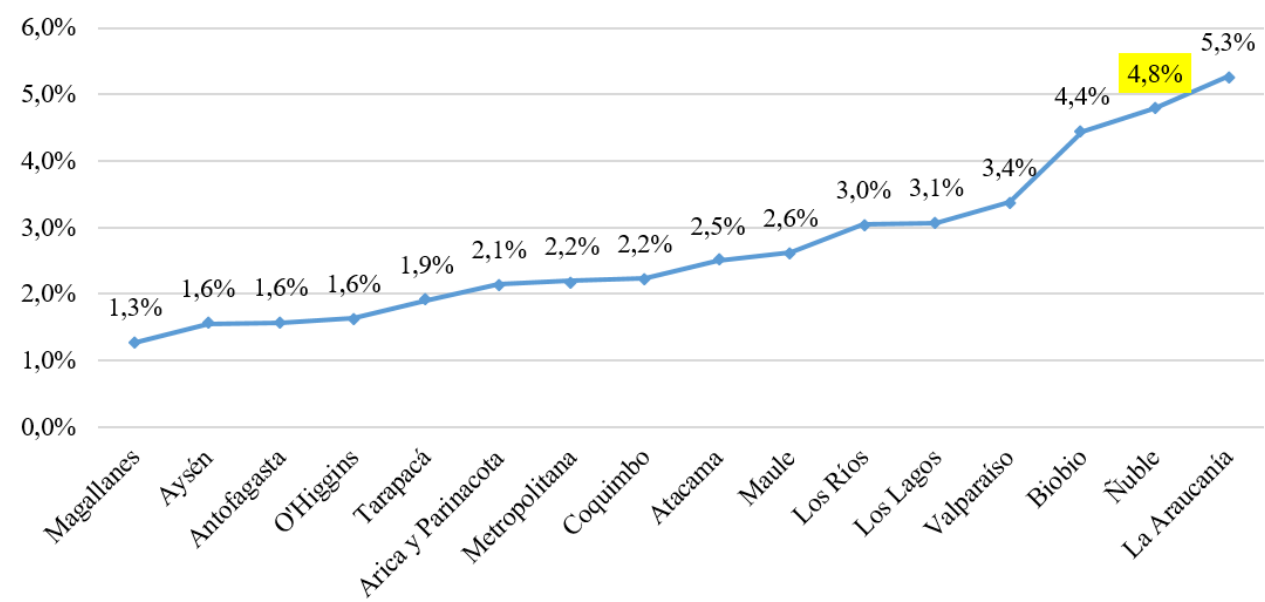

Fuente: elaboración propia con base en Encuesta CASEN 2011 
Tabla 1: Indicadores seleccionados para el análisis

\begin{tabular}{|c|c|c|}
\hline Categorías & Indicadores $^{3}$ & Elementos asociados \\
\hline \multirow{5}{*}{ Sociales } & Tasa de Pobreza & Desarrollo económico \\
\hline & Tasa de Indigencia & equilibrado \\
\hline & Nivel educacional & Aprovechamiento del capital territorial \\
\hline & Servicios básicos de la vivienda & Acceso equivalente a \\
\hline & Sistema de salud al que pertenece & equipamientos y servicios \\
\hline \multirow{4}{*}{ Económicas } & Condición de actividad & \\
\hline & Porcentaje de ocupados por sector & $\begin{array}{l}\text { Desartono economico } \\
\text { equilibrado }\end{array}$ \\
\hline & Ingreso promedio del hogar & \\
\hline & Acceso a internet & $\begin{array}{l}\text { Acceso equivalente a equipamientos y } \\
\text { servicios }\end{array}$ \\
\hline $\begin{array}{l}\text { Capacidades } \\
\text { institucionales }\end{array}$ & $\begin{array}{l}\text { Ingreso total percibido por municipalidades (prome- } \\
\text { dio por región) } \\
\text { Nivel de Profesionalización del Personal Municipal } \\
\text { Número de organizaciones sociales }\end{array}$ & $\begin{array}{l}\text { Aprovechamiento del } \\
\text { capital territorial }\end{array}$ \\
\hline \multirow{2}{*}{$\begin{array}{l}\text { Condiciones de } \\
\text { entorno }\end{array}$} & Cobertura de agua potable promedio por región & $\begin{array}{l}\text { Acceso equivalente a equipamientos y } \\
\text { servicios }\end{array}$ \\
\hline & $\begin{array}{l}\text { Metros cuadrados }\left(M^{2}\right) \text { de Áreas Verdes con Mante- } \\
\text { nimiento por (promedio por región) }\end{array}$ & $\begin{array}{l}\text { Acceso equivalente a equipamientos y } \\
\text { servicios }\end{array}$ \\
\hline
\end{tabular}

siendo superado por las regiones de Los Ríos (una de las últimas regiones creadas junto con la de Arica y Parinacota), Atacama, Maule, La Araucanía y Aysén.

- En cuanto a personal calificado de nivel superior (entendiendo como tal a técnicos de nivel superior o profesionales con formación completa), la futura región se ubica en el penúltimo lugar nacional, con un 6,1\% de personas con ese grado de formación, siendo ese porcentaje solo mejor que el que posee la región del Maule, que alcanza el 5,7\%.

Los datos señalados podrían ser una de las explicaciones de los altos niveles de pobreza e indigencia que presenta la zona y traen consigo un tema abordar para el eventual nuevo aparato regional, los cuales son el aumento de la escolaridad y de la capacitación y perfeccionamiento de los recursos humanos a nivel técnico y superior.

\section{Sistema de salud al que pertenece}

Los resultados obtenidos en materia de sistema de salud al que pertenecen los encuestados se presentan en la siguiente tabla:

Los datos presentados muestran la alta dependencia de la mayor parte de la población encuestada (50,5\%), del grupo A de FONASA, el cual incluye principalmente a personas indigentes o carentes de recursos, lo cual está en línea con lo analizado en los puntos anteriores sobre pobreza y escolaridad ${ }^{7}$. Esta participación es la más alta a nivel nacional, junto con la que se muestra en la región de La Araucanía.

Lo señalado anteriormente se complementa con la baja participación que existe de las personas de Nuble en las ISAPRES $(4,1 \%)$, el cual es el más bajo de todas las regiones.

\section{Indicadores económicos}

\section{Condición de actividad}

La condición de actividad a nivel regional se refleja en la figura 3 que se presenta a continuación:

De acuerdo a él, la zona de Nuble presenta un $49,2 \%$ de personas ocupadas y un 4,4\% de personas desocupadas (ambas cifras mejores que lo que sería la "nueva" región del Biobío), lo que está levemente

\footnotetext{
${ }^{7}$ Grupos de FONASA (información extraída del sitio Web http://www.supersalud.gob.cl/consultas/570/w3article-6304.html, visitada el 6 de noviembre de 2015):

- Tramo A: Personas indigentes o carentes de recursos; Beneficiarios de pensiones básicas solidarias; Causantes de subsidio familiar (Ley 18.020).

- Tramo B: Personas que perciben un ingreso imponible mensual menor o igual a $\$ 241.000$ pesos.

- Tramo C: Personas que perciben un ingreso imponible mensual mayor a $\$ 241.000$ pesos y menor o igual a $\$ 351.860$ pesos.
}

Tramo D: Personas que perciben un ingreso imponible mensual mayor a $\$ 351.860$ pesos. 
Tabla 2: Sistema de salud al que pertenecen, desagregado por región, año 2011

\begin{tabular}{lcc}
\hline \hline Regiones & $\tilde{N} u b l e$ & Promedio \\
\hline S. Público FONASA Grupo A & $50,50 \%$ & $34,20 \%$ \\
S. Público FONASA Grupo B & $28,20 \%$ & $27,20 \%$ \\
S. Público FONASA Grupo C & $9,00 \%$ & $10,90 \%$ \\
S. Público FONASA Grupo D & $4,50 \%$ & $7,60 \%$ \\
S. Público FONASA no sabe grupo & $0,70 \%$ & $2,90 \%$ \\
F.F.A.A. y del Orden & $2,20 \%$ & $2,50 \%$ \\
ISAPRE & $4,10 \%$ & $10,20 \%$ \\
Ninguno (particular) & $0,30 \%$ & $2,80 \%$ \\
Otro sistema & $0,20 \%$ & $0,50 \%$ \\
No sabe & $0,40 \%$ & $1,20 \%$ \\
\hline Total & $100 \%$ & $100 \%$ \\
\hline \hline Fuente: elaboración propia con base en Encuesta CASEN 2011
\end{tabular}

Figura 3: Condición de actividad por región, año 2011 (en porcentajes)

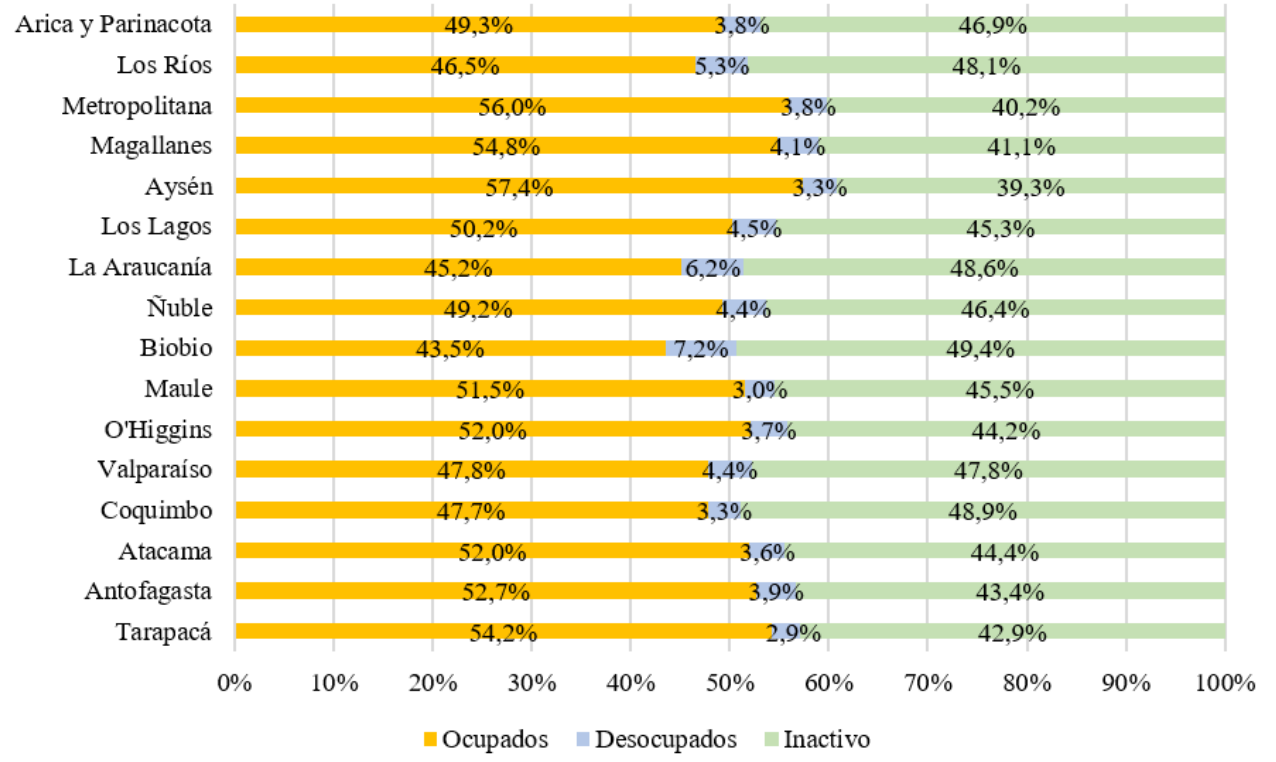

Fuente: elaboración propia con base en Encuesta CASEN 2011

por debajo de los promedios nacionales, que llegan a $50,6 \%$ y a $4,2 \%$, respectivamente.

En cuanto a los inactivos, estos llegan a $46,4 \%$ en Ñuble, lo que está levemente por sobre el promedio nacional que alcanza el $45,1 \%$.

\section{Porcentaje de ocupados por sector}

Un indicador relacionado a los anteriormente analizados es el que muestra el porcentaje de ocupados distribuido para cada uno de los sectores de la economía. Los resultados de este indicador se presentan en la figura 4:

A partir de la figura anterior se puede constatar que el sector terciario (servicios) representa la mayor participación en la futura región de Nuble (aunque el promedio nacional llega a 63,3\%) y, en general, en todas las regiones del país. Además, se destaca la participación del sector primario en la ocupación de dicha zona, siendo una de las mayores a nivel nacional (es más que el promedio nacional que llega al 18,4\%), siendo superada por las regiones del Maule, O'Higgins y Coquimbo.

Por último, la menor participación la presenta el sector secundario, con un porcentaje que prácticamente coincide con la media nacional, que llega a $\operatorname{los} 18,3 \%$. 
Figura 4: Ocupados por sector productivo, desagregado por región, año 2011 (en porcentajes)

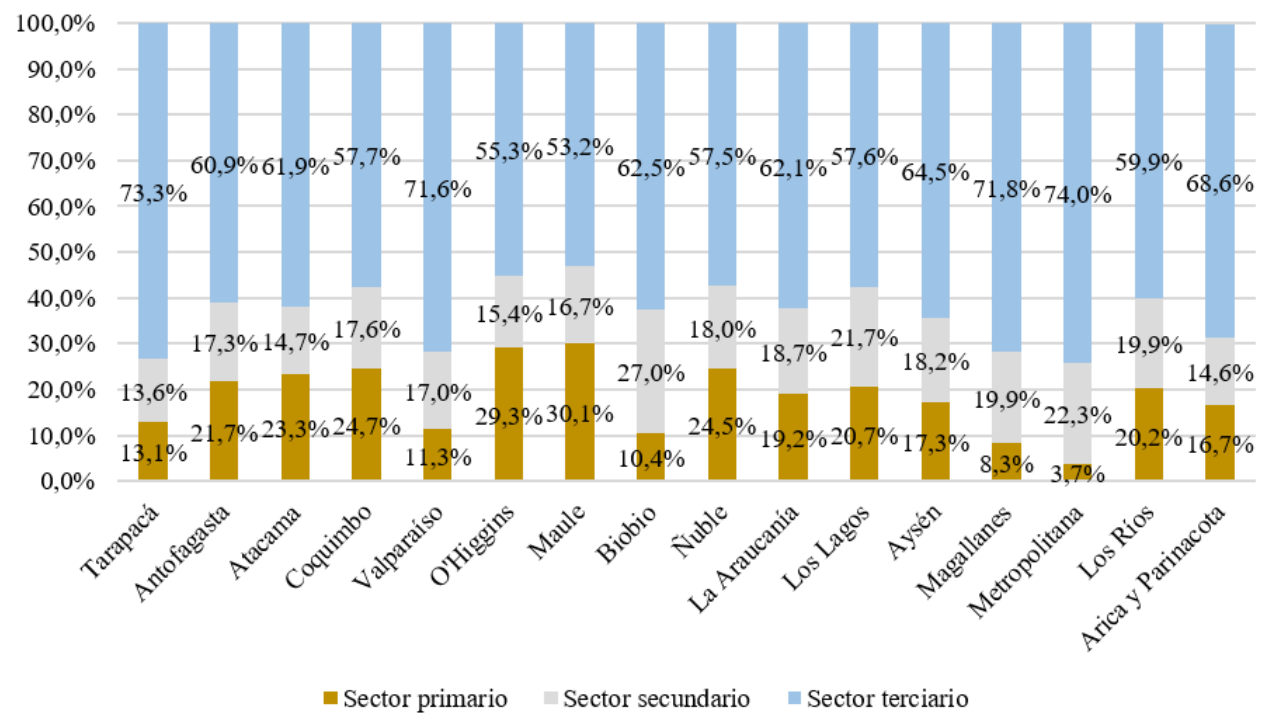

Fuente: elaboración propia con base en Encuesta CASEN 2011

\section{Ingreso promedio del hogar}

El ingreso promedio de los hogares, desagregado por región, se presenta en la figura $5^{8}$

Al respecto, destaca Nuble por presentar un ingreso mayoritariamente bajo por hogar, teniendo en este nivel casi la mayor participación a nivel nacional (detrás de la región de La Araucanía), siendo el promedio nacional de $21,1 \%$.

Por su parte, el promedio general de los hogares de Nuble es de $\$ 473.080$, lo que es menos de la mitad de la media nacional, que asciende a los $\$ 1.060 .157$.

\section{Capacidades institucionales}

Las capacidades institucionales se centran en la situación de las municipalidades de cada región, lo que se analiza a partir de las siguientes variables:

\footnotetext{
${ }^{8}$ Los tramos de ingreso fueron definidos para efectos de este trabajo, buscando generar 5 grupos iguales, por percentiles de ingreso. Los montos asociados a cada uno de ellos son los siguientes:

- Ingreso bajo: valor más bajo a $\$ 299.603$

- Ingreso medio bajo: $\$ 299.604$ a $\$ 475.130$

- Ingreso medio: $\$ 475.131$ a $\$ 710.116$

- Ingreso medio alto: $\$ 710.117$ a $\$ 1.193 .898$

- Ingreso alto: $\$ 1.193 .899$ a valor más alto
}

\section{Ingreso total percibido por municipalidades}

De acuerdo con los antecedentes extraídos del SINIM, para el año 2014, el ingreso total percibido debe entenderse como el conjunto de recursos percibidos por cada municipalidad durante el periodo fiscal, el cual se comprende entre los meses de enero y diciembre. En este caso, corresponde al ingreso total promedio de las municipalidades, agrupado por región.

Teniendo en consideración lo señalado, de acuerdo con lo mostrado por la figura 6, las municipalidades de Nuble presentan de los más bajos ingresos totales promedio a nivel nacional, superando solo al promedio de las municipalidades de Aysén.

Por su parte, también destaca que su cifra está por bajo el promedio nacional, que llega a los M\$8.417.068. Sobre esto, no obstante, es importante consignar la importante influencia que ejerce en él el promedio que presentan las municipalidades de la región Metropolitana, el cual escapa a las medias mostradas por el resto de las regiones.

\section{Nivel de Profesionalización del Personal Mu- nicipal}

El nivel de profesionalización del personal municipal, siguiendo lo disponible en el SINIM, recoge la proporción de recursos humanos que dispone cada municipalidad que tiene la calificación de "profesional". Para este trabajo, ese dato corresponde al nivel de profesionalización promedio que presenta el personal municipal de cada región. 
Figura 5: Ingreso promedio por hogar de cada región, año 2011 (en porcentajes)

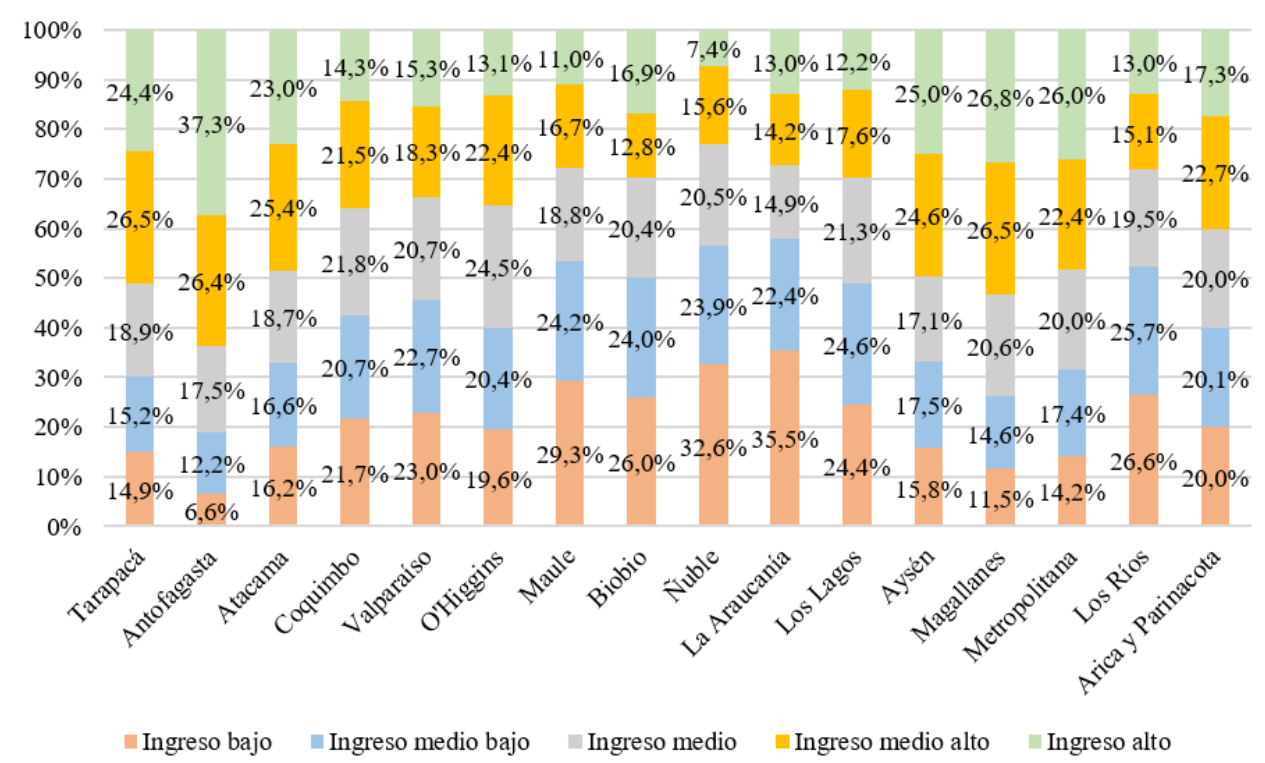

Fuente: elaboración propia con base en Encuesta CASEN 2011

Figura 6: Ingreso total promedio de las municipalidades, agrupado por región, año 2014 (en M\$)

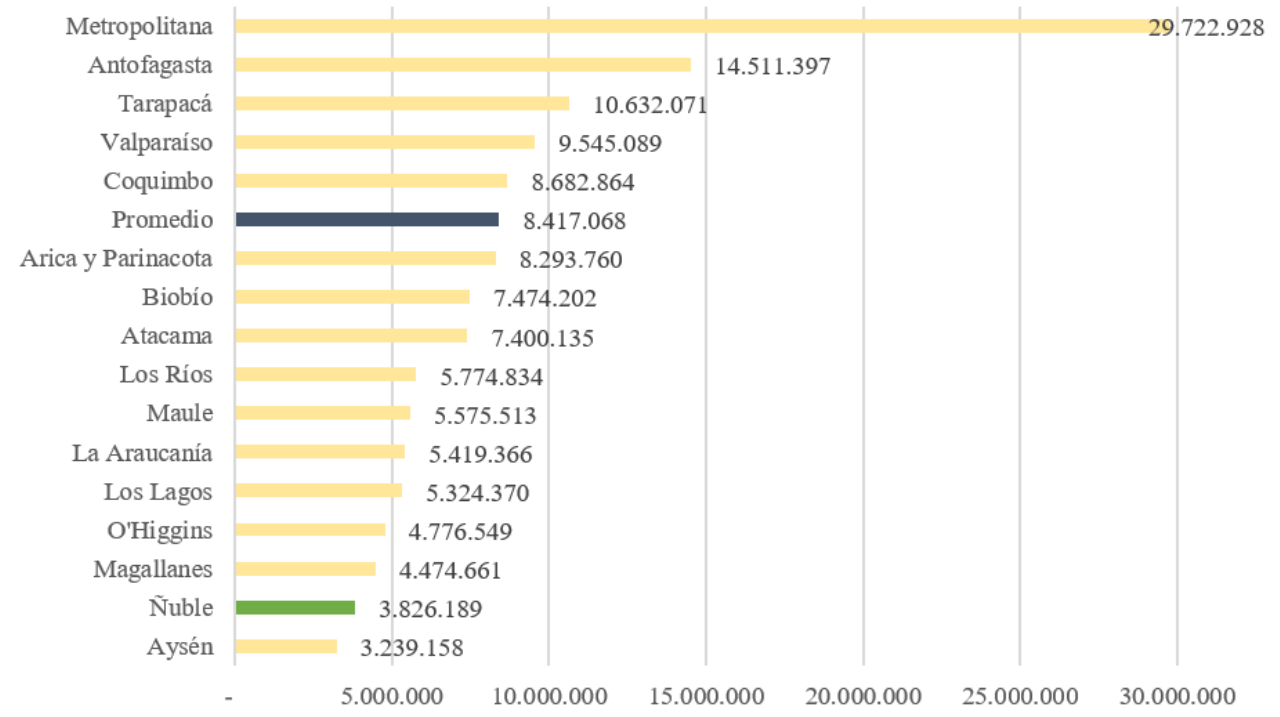

Fuente: elaboración propia con base en SINIM 2014 
Figura 7: Nivel promedio de Profesionalización del Personal Municipal, por región, año 2014 (en porcentajes)

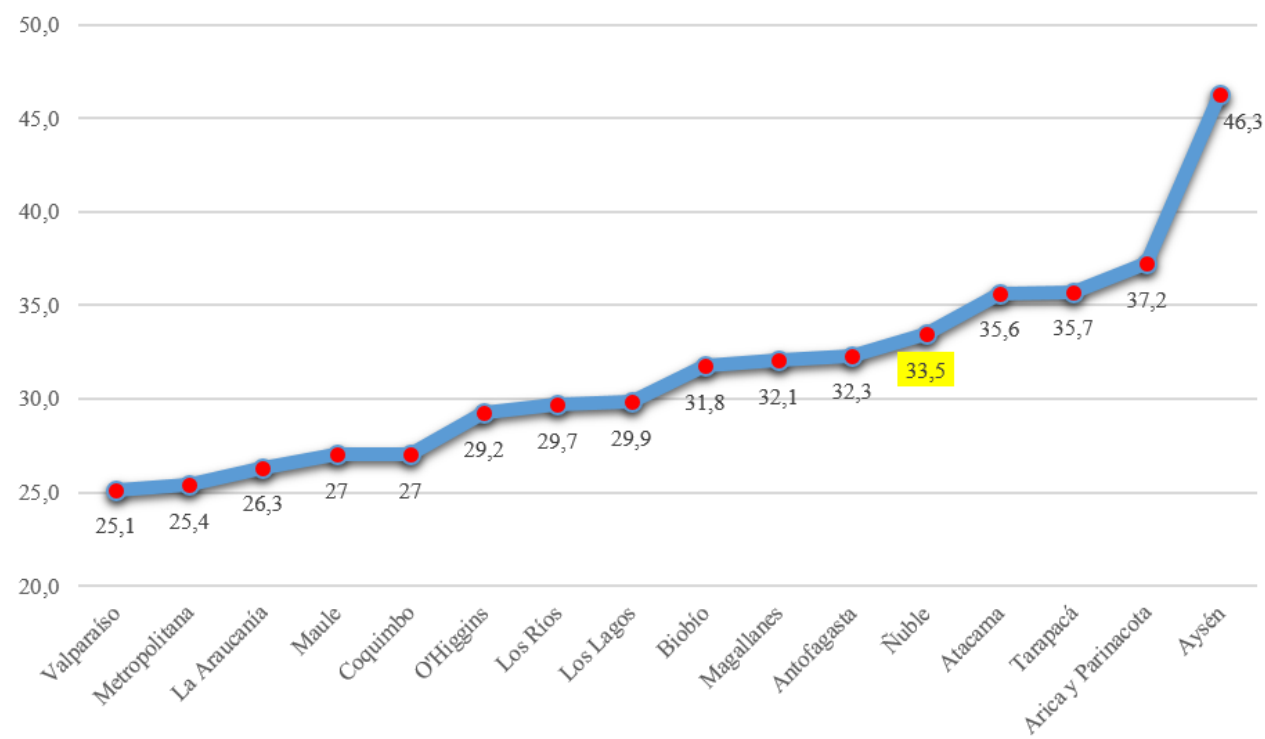

Fuente: elaboración propia con base en SINIM 2014

Respecto a los resultados obtenidos, estos se contienen en la figura 7. A partir de ellos, se constata que la situación de Nuble es positiva a nivel regional, con un 33,5\% de profesionalización, la cual la posiciona en el tercio más alto a nivel regional.

Por su parte, la media de profesionalización alcanza al $31,5 \%$, por lo que las municipalidades de Nuble también están por sobre ese indicador.

Por último, es importante señalar que se espera que esta profesionalización mejore en general a partir la ley que busca mejorar las condiciones laborales en las municipalidades a través de diversas vías, el cual actualmente está en su proceso final de tramitación ${ }^{9}$.

\section{Número de organizaciones sociales}

El número de organizaciones sociales se analiza como una medida aproximada de magnitud con la que se da la participación ciudadana (incluye a centros de madres, centros de padres y apoderados, centros $\mathrm{u}$ organizaciones del adulto mayor, clubes deportivos, juntas de vecinos, uniones comunales y a otras organizaciones comunitarias funcionales).

La región del Nuble tiene un total de 6.919 organizaciones sociales, concentradas principalmente en organizaciones comunitarias funcionales. Esto la ubica en la mitad del cuadro en relación al resto de las regiones, estando por debajo de lo que sería la

\footnotetext{
${ }^{9}$ Ley $\mathrm{N}^{\circ} 20.922$ que "Modifica disposiciones aplicables a los funcionarios municipales y entrega nuevas competencias a la Subsecretaría de Desarrollo Regional y Administrativo".
}

"nueva" región del Biobío.

En cuanto a organismos de representación con mayor componente político, como lo son las juntas de vecinos y las uniones comunales, la futura región presenta cantidades que la dejan dentro del segmento de regiones que tienen un mayor número de ellas. Esto podría ser interpretado como un indicador de que existen en la zona prácticas de participación ciudadana, a través de organismos como los señalados, que podrían ser de utilidad para impulsar acciones en materia de cohesión territorial.

\section{Condiciones de entorno}

\section{Cobertura de agua potable promedio por re- gión}

Los resultados del indicador de cobertura de agua potable, definidos a partir del promedio que presentan las comunas que conforman cada región se muestran en la tabla 3 .

En este indicado, la zona de Nuble se ubica dentro de las regiones que presenta los menores niveles de cobertura, en relación al resto de las regiones, alcanzando solo un 55,97\%, con lo cual supera únicamente a las regiones de Magallanes, de Los Lagos y de Arica y Parinacota. Además, es importante señalar que la situación de la que sería la región del Biobío sería mejor que la de Nuble en alrededor de un $20 \%$.

Junto con ello, es importante destacar que existen varias comunas dentro de la futura región que 
Tabla 3: Porcentaje promedio de cobertura de agua potable, por región, año 2014

\begin{tabular}{ll}
\hline \hline Regiones & \multicolumn{2}{l}{$\begin{array}{l}\text { Cobertura de } \\
\text { Agua }\end{array}$} \\
(\%) & Potable \\
\hline Antofagasta & 95,33 \\
Metropolitana & 94,60 \\
Atacama & 91,43 \\
Valparaíso & 88,52 \\
O'Higgins & 82,78 \\
Coquimbo & 81,66 \\
Aysén & 79,83 \\
Biobío & 75,28 \\
Maule & 71,88 \\
Tarapacá & 71,67 \\
Los Ríos & 62,06 \\
La Araucanía & 59,59 \\
Ñuble & $\mathbf{5 5 , 9 7}$ \\
Magallanes & 54,53 \\
Los Lagos & 52,96 \\
Arica y Parinacota & 48,26 \\
\hline
\end{tabular}

Fuente: Elaboración propia con base en SINIM 2014

presentan coberturas por debajo del $50 \%$, tales como Cobquecura, El Carmen, Ninhue, Pinto, Portezuelo, Quillón, San Ignacio, San Nicolás y Trehuaco, cuyo promedio solo llega al $38,79 \%$.

\section{Metros cuadrados $\left(M^{2}\right)$ de Áreas Verdes con Mantenimiento por Habitante (prome- dio por región)}

En lo que se refiere a metros cuadras de áreas verdes por habitante, el promedio por región se señala en el cuadro 5 .

Respecto a lo que muestra el cuadro ya indicado, al igual que en caso anterior, la región del Ñuble está dentro de las que presenta los peores resultados, con solo 2,79 $\mathrm{m} 2$ por habitantes, lo que es similar la región del Maule y superior a la región de Tarapacá, que son las únicas que están por debajo del territorio en análisis.

Además de lo expuesto, es posible señalar que las comunas que conformarían la nueva región presentan un rango en este indicador que fluctúa entre los 0,4 m2 (comuna de Pinto) y los 5,29 m2 (comuna de San Nicolás).

\section{Conclusiones y recomendacio- nes de política pública}

A partir de los resultados presentados en el punto anterior se puede concluir que, en general, lo que sería la futura región de Nuble presenta una situación de rezago y debilidad de su nivel y condiciones de desarrollo territorial respecto a la mayoría de las regiones del país en los ámbitos económicos y sociales analizados, lo cual es coherente con parte de los planteamientos que han presentado los grupos que están apoyando la creación de esta nueva identidad territorial.

Esto se expresa también en varios indicadores, si se compara la zona de la nueva región en relación a lo que sería la "nueva" región del Biobío, comprendida por las provincias de Concepción, Biobío y Arauco.

Por su parte, para abordar la serie de brechas que fueron analizadas a lo largo de este trabajo, así como también otras relacionadas que no fueron parte de él, pero que sí se han documentado en estudios tales como el realizado por la Universidad de Concepción (que fue citado anteriormente) y, más recientemente, el efectuado por la Universidad del Biobío ${ }^{10}$, la zona de Nuble dispone de

\footnotetext{
${ }^{10}$ Denominado "Caracterización de la Provincia de Nuble y una propuesta estratégica para el desarrollo del territorio". Antecedentes sobre este trabajo están disponibles en el siguiente sitio Web http://noticias.ubiobio.cl/2015/10/30/academicosubb-presentan-libro-con-mirada-estrategica-para-el-
} 
Tabla 4: Metros cuadrados de áreas verdes por habitante (promedio)

\begin{tabular}{ll}
\hline \hline Regiones & $\begin{array}{l}M t^{2} \text { área verde / } \\
\text { habitante }\end{array}$ \\
\hline Aysén & 35,18 \\
Magallanes & 9,81 \\
Arica y Parinacota & 8,33 \\
Los Lagos & 6,02 \\
La Araucanía & 4,48 \\
O'Higgins & 3,93 \\
Valparaíso & 3,88 \\
Los Ríos & 3,64 \\
Metropolitana & 3,59 \\
Biobío & 3,16 \\
Atacama & 2,85 \\
Coquimbo & 2,82 \\
Antofagasta & 2,81 \\
Ñuble & $\mathbf{2 , 7 9}$ \\
Maule & 2,57 \\
Tarapacá & 1,57 \\
Fuente: Elaboración propia con base en SINIM 2014
\end{tabular}

algunas condiciones que pueden ser favorables, tales como el nivel de profesionalización de presentan sus municipalidades. Esto puede verse potenciado a partir de la puesta en marcha de modificaciones legales recientemente aprobadas y otras actualmente en discusión que, en general, apuntan a profundizar los procesos de descentralización y a potenciar y ampliar las capacidades que disponen los organismos subnacionales para ser sujetos activos de su desarrollo ${ }^{11}$.

Todo esto trae consigo que, desde la perspectiva de la cohesión territorial como un objetivo de la creación de esta nueva región (en el contexto de la definición propuesta anteriormente, la cual la entiende tanto como una condición como a una capacidad local mediante la cual todas las zonas de un determinado lugar están en posición de disponer y generar niveles mínimos de bienestar, definidos como tal por la sociedad reconociendo los rasgos particulares que implican para cada sector), ello se podría justificar si es que el aparato regional permitirá abordar las brechas, lo cual implica una serie de desafíos para la institucionalidad subnacional actual. Entre ellos, siguiendo lo que propuesto por Candia (2015), es posible mencionar los siguientes:

- Generar un sistema regional de planificación con foco territorial.

\footnotetext{
desarrollo-de-nuble/, visitado el 9 de noviembre de 2015.

${ }^{11}$ Entre ellas están, además de la citada respecto al mejoramiento de las condiciones laborales a nivel municipal, la elección directa de los intendentes, el traspaso de competencias en temas de fomento productivo, desarrollo social e infraestructura y transporte y el mejoramiento de los mecanismos de participación ciudadana, entre otras.
}

- Definir cuotas territoriales de la inversión pública regional, que eviten a priori la concentración de ella en la provincia (o a la ciudad) que acogerá a la capital regional y que permitan promover un desarrollo del conjunto del territorio de la nueva región.

- Generar criterios complementarios a los existentes para evaluar proyectos de inversión pública basados, por ejemplo, en la creación de una especie de "rentabilidad territorial" que sea capaz de valorar en los proyectos indicadores que den cuenta de los efectos específicos que se generarán en un determinado lugar y su grado de relación con las carencias que presentan en relación a sus objetivos de desarrollo.

- En la lógica de impulsar un enfoque territorial del desarrollo de la región, se requiere fortalecer las municipalidades de Ñuble, de manera que todas puedan ser agentes protagónicos de dicho proceso.

Además, para mejorar la focalización de la política pública es importante conocer y reconocer las particularidades intrarregionales, las que pueden implicar diferencias considerables de la situación y capacidades que presente cada localidad, sobre todo en una zona como la de Ñuble que tiene un porcentaje importante de población en zonas rurales. 


\section{Recomendaciones de política pública}

Son diversas las implicancias que tiene la inclusión del enfoque de cohesión territorial en los procesos de políticas públicas. Entre las principales están las siguientes:

1. Siguiendo a RIMISP (2013), es necesario estructurar y dar la coherencia a las intervenciones bajo una sola estrategia que contemple intervenciones en 4 niveles:

- Políticas sectoriales sensibles a las diferencias territoriales: que evitan, reducen o compensan los efectos negativos que producen las intervenciones sectoriales en materia de desigualdad.

- Políticas sectoriales de reducción de brechas: que se orientan a equiparar las condiciones y oportunidades de desarrollo a lo largo del territorio.

- Políticas de descentralización: que transfieren poder, competencias, funciones y recursos en los ámbitos político, administrativo y fiscal a las entidades subnacionales.

- Políticas de desarrollo territorial: para promover y potenciar las condiciones y ventajas comparativas propias de cada lugar, generando las capacidades necesarias para ello.

2. En esa perspectiva, tareas como la coordinación y coherencia de la acción sectorial con la territorial aparece como primordiales, dada la mayor complejidad que implica para las intervenciones de política pública la estructura propuesta anteriormente. Para ello, de acuerdo con ODTA (2009), para mejorar esa coordinación es posible utilizar las siguientes vías:

- "Territorialización" de las políticas públicas, es decir, que se contemplen en su diseño las formas (el cómo y el dónde) y las implicancias directas e indirectas que tendrá en su implementación en cada lugar.

- Vinculación de las intervenciones a una estrategia de desarrollo territorial claramente definida, ya que si la acción de política se desprende de instrumentos de planificación territorial, todas las acciones que se generen a partir de ella deberían tener una justificación y coherencia clara, de acuerdo también a los ámbitos de competencia de cada uno de sus ejecutores. Esto, por cierto, exige que tales instrumentos sean de calidad, cuyas implicancias serán analizadas más adelante.

- Establecimiento de una eficaz gobernanza territorial que sea capaz de "coordinar enfoques, políticas y actores sociales diversos, [y que cuente con] algún tipo de "autoridad" o entidad de coordinación de alto rango ODTA, 2009". Al respecto, es especialmente importante contar con mecanismos de arbitraje y resolución de controversias entre entidades lo que, a su vez, requiere de una institucionalidad adecuada para ello.

Además de lo ya señalado, siguiendo el trabajo de la ODTA, también puede ser de utilidad para la coordinación y la coherencia de las políticas el disponer de adecuadas herramientas de información y caracterización de los territorios (con datos espacialmente desagregados que permitan efectuar análisis focalizados en materias tales como estado actual, brechas, acciones en ejecución, resultados obtenidos por ellas, etc.).

3. Generación de mecanismos de gobernanza y de participación: Tal como se ha señalado en puntos anteriores, la complejidad de la acción de política pública ya analizada también se origina a partir de la necesidad de disponer de más y mejores mecanismos de participación ciudadana (que van desde mecanismos vinculantes de consulta hasta espacios permanentes de diálogo en torno a temas relevantes para el desarrollo territorial).

Al respecto, la ODTA (2009) destaca que "es evidente que el contexto actual parece requerir nuevas formas de gestión sociopolítica que respondan de manera más adecuada y eficiente a los retos que se plantean. Dado que las viejas estructuras políticas (el Estado fundamentalmente) parecen necesitar una reformulación, frente al papel de nuevos actores sociales (grupos financieros transnacionales, ONGs, grupos científicos, etc.), no parece descabellado pensar en formas organizativas alternativas donde primen la flexibilidad, la adaptabilidad, la cooperación entre actores, la participación activa de todos ellos, y la autonomía y la creatividad en la toma de decisiones" (ODTA, 2009: 9).

Por su parte, Silva y Echeverri (2015), plantean que "como consecuencia de la transición institucional, la participación de las instancias territoriales, de la sociedad civil y del sector privado es crucial para alcanzar el objetivo de 
desarrollo armónico propuesto desde la perspectiva de la cohesión territorial, en cada uno de los niveles y escalas, y entre ellos" (Silva y Echeverri, 2015: 37). Esta participación, de acuerdo con ambos autores, se debe orientar hacia la formulación, promoción y defensa de los proyectos territoriales de desarrollo y el fortalecimiento y preservación de la identidad territorial común.

4. Formular estrategias de desarrollo: como instrumentos de base de la acción pública, privada y de la sociedad territorial en general, establecidos a partir de diagnósticos, objetivos y acciones consensuadas por todos ellos, de los que además se derivan compromisos de aportes concretos, generando con ello corresponsabilidad de los involucrados en el logro de los propósitos definidos.

Además, estos instrumentos, teniendo como eje el logro de cohesión territorial, "[...] permiten la articulación de la eficiencia económica, cohesión social, equilibrio ecológico y gobernanza, en un entorno concreto de carácter integral en un sistema territorial. Con ello se logra tener pautas y orientaciones más claras para las decisiones necesarias en el logro de la equidad en la distribución de servicios, equipamiento e infraestructura, es decir, establece un principio de ordenamiento del territorio" (Silva y Echeverri, 2015: 36).

5. Necesidad de generar capacidades institucionales: todo lo anterior requiere de capacidades institucionales (con personas calificadas, organización, recursos, funciones y atribuciones) asentadas localmente, de tal forma de que los actores de ese nivel asuman el protagonismo de su proceso de desarrollo. Esto se complementa con lo expuesto por Silva y Echeverri (2015), quienes señalan que "[...] la cohesión territorial [...] implica requerimientos específicos de capacidad institucional para la promoción y regulación de los procesos de integración de los sistemas territoriales [...]. [Se] requiere de una institucionalidad capaz de actuar desde y sobre los territorios [...]" (Silva y Echeverri, 2015: $37)$.

Otro requerimiento que se deriva de la acción pública con foco en la cohesión territorial es el relacionado con conectividad, entendiendo como tal (siguiendo a Silva y Echeverri (2015)) a las vías físicas y virtuales que existen para la articulación entre los distintos componentes del sistema territorial que representa un factor que incluye en la forma e intensidad de las relaciones funcionales entre los actores distribuidos a lo largo de cada territorio.
Además, las características de esta conectividad influyen en la forma en que se ocupa y aprovecha el territorio.

Por último, dada la diversidad de desafíos que fueron señalados anteriormente, es conveniente centrarse en aquellas acciones que permitan generar las condiciones necesarias para posibilitar la legitimidad y sustentabilidad de los procesos de desarrollo que se impulsarán y las medidas asociadas a su consecución. En ese sentido, las tareas señaladas, ligadas a gobernanza y participación ciudadana y formulación de estrategias de desarrollo aparecen como primordiales, junto con la generación de capacidades institucionales asociadas.

\section{Referencias}

Candia, R. (2015). Ideas para ñuble región. columna de opinión. disponible en http://www.elquintopoder.cl/desarrolloregional/ideas-para-nuble-region/.

CRMP (2008). Cohesión territorial - elementos para enfocar el concepto y hacerlo operativo. Nota técnica de la Secretaría General de la Conferencia de Regiones Periféricas Marítimas de Europa.

Neumark, D. y Simpson, H. (2014). Place-based policies. Technical report, National Bureau of Economic Research.

ODTA (2009). Aportaciones del Foro de expertos sobre la cohesión, la diversidad y el desarrollo territorial. Reflexiones en torno al libro verde de la Comisión de las Comunidades Europeas. Observatorio de la Diversidad, Cohesión y el Desarrollo Territorial de Andalucía.

Pillet, F., Canizares, M., Ruiz, A., Martínez, H., Plaza, J., y Santos, J. (2013). Los indicadores de la cohesión territorial en el análisis de la escala supramunicipal o subregional: policentrismo y áreas funcionales urbanas (fuas). ERIA Revista cuatrimestral de geografía, (90):91-106.

Rentería, M. T. (2011). Tipos y modos de la participación ciudadana en los procesos de ordenación del territorio. dos casos para su ilustración. Ordenamiento territorial y participación social: problemas y posibilidades, pp. 447-470.

RIMISP (2013). Nueva agenda regional contra la desigualdad. En Seminario Nueva Agenda Regional.

Schejtman, A. y Berdegué, J. (2004). Desarrollo territorial rural. Debates y temas rurales, 1:7-46. 
Silva, I. y Echeverri, R. (2015). Estrategias y políticas nacionales para la cohesión territorial: estudios de caso latinoamericanos. Technical report, Naciones Unidas Comisión Económica para América Latina y el Caribe (CEPAL).

Tabales, A. F., Mateos, B. P., Mateos, J. C. R.,
López, M. F. P., y Naranjo, F. Z. (2009). El concepto de cohesión territorial: escalas de aplicación, sistemas de medición y políticas derivadas. Boletín de la Asociación de Geógrafos Españoles, (50):157-172. 